\title{
Measured and Simulated Motion of a Hopping Rotochute
}

\author{
Eric Beyer* and Mark Costello£ \\ Georgia Institute of Technology, Atlanta, Georgia 30332 \\ DOI: $10.2514 / 1.41331$
}

\begin{abstract}
A new hybrid micro vehicle called the hopping rotochute has been developed to robustly explore rough and complex terrain. Unlike other jumping robots, the vehicle traverses an area by intermittently powering a small coaxial rotor system that allows the device to hop over obstacles of various shapes and sizes. A movable internal mass controls the vehicle's direction of travel, and the exterior shape and low mass center allow the vehicle to passively reorient itself to an upright attitude when in contact with the ground. This paper presents a dynamic model of the hopping rotochute used to assess the basic flight performance of the vehicle. The experimental methods used to estimate model parameters are described and comparisons between measured and simulated motion are presented. Basic flight performance predicted by dynamic simulation is reported, including maneuverability, jumping performance, and total range. The simulated results indicate that the hopping rotochute is capable of surmounting larger obstacles than current hoppers while being able to navigate with the use of a movable internal mass.
\end{abstract}

\begin{tabular}{|c|c|}
\hline & Nomenclature \\
\hline$C_{D}$ & $=$ aerodynamic drag coefficient \\
\hline$C_{l p}, C_{m q}, C_{n r}$ & $=$ aerodynamic damping moment coefficients \\
\hline$c_{1 n}, c_{1 t}$ & $\begin{aligned}= & \text { normal and tangential damping coefficients } \\
& \text { associated with body vertices }\end{aligned}$ \\
\hline$c_{2 n}, c_{2 t}$ & $\begin{aligned}= & \text { normal and tangential damping coefficients } \\
& \text { associated with the ground face }\end{aligned}$ \\
\hline$D$ & $=$ aerodynamic reference diameter \\
\hline $\begin{array}{l}\vec{F}_{n}, \vec{F}_{t} \\
d_{\mathrm{IM}}\end{array}$ & $\begin{array}{l}=\text { normal and tangential contact force vectors } \\
=\text { internal mass offset parameter }\end{array}$ \\
\hline$g$ & $=$ acceleration of gravity \\
\hline$I$ & $\begin{aligned}= & \text { inertia matrix of the hopping rotochute } \\
& \text { about the mass center }\end{aligned}$ \\
\hline$k_{1 n}, k_{1 t}$ & $\begin{aligned}= & \text { normal and tangential spring constants } \\
& \text { associated with body vertices }\end{aligned}$ \\
\hline$k_{2 n}, k_{2 t}$ & $\begin{aligned}= & \text { normal and tangential spring constants } \\
& \text { associated with the ground face }\end{aligned}$ \\
\hline$L, M, N$ & $\begin{aligned}= & \text { applied moment components about the mass } \\
& \text { center in the body reference frame }\end{aligned}$ \\
\hline$m$ & $=$ mass of the hopping rotochute \\
\hline$p, q, r$ & $\begin{array}{l}=\text { components of angular velocity vector in the } \\
\text { body reference frame }\end{array}$ \\
\hline $\mathbf{R}_{A \rightarrow B}$ & $\begin{aligned}= & \text { skew-symmetric matrix representation of the } \\
& \text { position vector from a generic point } A \text { to } \\
& \text { another point } B \text { in the body reference frame }\end{aligned}$ \\
\hline$S$ & $=$ aerodynamic reference area \\
\hline $\begin{array}{l}\mathrm{SL}_{A \rightarrow B}, \mathrm{BL}_{A \rightarrow B}, \\
\mathrm{WL}_{A \rightarrow B}\end{array}$ & $\begin{aligned} &= \text { components of the position vector from a } \\
& \text { generic point } A \text { to another point } B \text { in the } \\
& \text { body reference frame along the station line, } \\
& \text { butt line, and waterline }\end{aligned}$ \\
\hline$\vec{s}_{1 n}, \vec{s}_{1 t}$ & $\begin{aligned}= & \text { normal and tangential spring distance } \\
& \text { vectors associated with body vertices }\end{aligned}$ \\
\hline$\vec{s}_{2 n}, \vec{s}_{2 t}$ & $\begin{aligned}= & \text { normal and tangential spring distance } \\
& \text { vectors associated with the ground face }\end{aligned}$ \\
\hline$T$ & $=$ rotor thrust \\
\hline
\end{tabular}

Received 30 September 2008; accepted for publication 11 February 2009. Copyright (c) 2009 by Eric Beyer and Mark Costello. Published by the American Institute of Aeronautics and Astronautics, Inc., with permission. Copies of this paper may be made for personal or internal use, on condition that the copier pay the $\$ 10.00$ per-copy fee to the Copyright Clearance Center, Inc., 222 Rosewood Drive, Danvers, MA 01923; include the code 0731-5090/ 09 and $\$ 10.00$ in correspondence with the CCC.

${ }^{*}$ Graduate Research Assistant, School of Aerospace Engineering, Member AIAA.

${ }^{\dagger}$ Sikorsky Associate Professor, School of Aerospace Engineering. Associate Fellow AIAA.

\begin{tabular}{|c|c|c|}
\hline $\mathbf{T}_{\mathrm{BI}}$ & $=$ & $\begin{array}{l}\text { transformation matrix from the inertial } \\
\text { reference frame to the body reference frame }\end{array}$ \\
\hline $\mathbf{T}_{\mathrm{BR}}$ & $=$ & $\begin{array}{l}\text { transformation matrix from the rotor } \\
\text { reference frame to the body reference frame }\end{array}$ \\
\hline $\mathbf{T}_{\mathrm{IB}}$ & $=$ & $\begin{array}{l}\text { transformation matrix from the body } \\
\text { reference frame to the inertial reference } \\
\text { frame }\end{array}$ \\
\hline$u, v, w$ & $=$ & $\begin{array}{l}\text { components of the mass center velocity } \\
\text { vector in the body reference frame }\end{array}$ \\
\hline$\vec{u}$ & $=$ & absolute velocity of the contact point \\
\hline$\vec{w}$ & $=$ & absolute velocity of the surface elements \\
\hline$X, Y, Z$ & $=$ & $\begin{array}{l}\text { applied force components in the body } \\
\text { reference frame }\end{array}$ \\
\hline$x, y, z$ & $=$ & $\begin{array}{l}\text { components of the mass center position } \\
\text { vector in an inertial frame }\end{array}$ \\
\hline$\mu$ & $=$ & coefficient of friction \\
\hline$\rho$ & $=$ & density of air \\
\hline$\tau$ & $=$ & rotor lag time constant \\
\hline$\phi, \theta, \psi$ & $=$ & $\begin{array}{l}\text { Euler roll, pitch, and yaw angles of the } \\
\text { hopping rotochute }\end{array}$ \\
\hline$\phi_{R}, \theta_{R}$ & $=$ & $\begin{array}{l}\text { Euler roll and pitch angles of the rotor tip } \\
\text { path plane }\end{array}$ \\
\hline$\psi_{\mathrm{IM}}$ & $=$ & internal mass orientation parameter \\
\hline
\end{tabular}

\section{Introduction}

$\mathbf{M}$ ANY different types of micro vehicle configurations have been developed to aid in the exploration of irregular terrain such as damaged buildings and caves. Ground vehicles equipped with wheels or legs have limited success in these complex environments when large obstacles or gullies are encountered. Micro air vehicles, on the other hand, are not hindered by large obstructions, but have limited endurance due to the fact that they must remain airborne during the entire mission. To help alleviate these limitations, micro hybrid vehicles have been developed that are able to hop over obstacles much greater than their size and can loiter on the ground to preserve energy. The two-wheeled Scout, for example, rolls over smooth terrain and uses a spring foot to propel itself up to $35 \mathrm{~cm}$ into the air to negotiate uneven terrain [1]. The Jumping Mini-Whegs, on the other hand, is able to traverse using 4 wheel-like legs and is able to jump up to $22 \mathrm{~cm}$ with the use of a four-bar linkage and coil spring $[2,3]$. A series of hoppers developed by the Jet Propulsion Laboratory and California Institute of Technology are based on a six-bar linkage and coil-spring leg mechanism that can elevate the vehicles up to $1.2 \mathrm{~m}$ high [4]. Yet another hopper, developed at Sandia National Laboratories, uses the combustion of liquid propane to drive a piston into the ground, allowing it to reach altitudes of $1 \mathrm{~m}[\underline{5}, 6]$. 
A new hybrid micro vehicle configuration called the hopping rotochute has been developed to robustly operate within irregular terrain while minimizing energy consumption. Unlike the other hopping robots previously described, the hopping rotochute is propelled upward by a motor-driven coaxial rotor system. The motor is powered in bursts, allowing the vehicle to hop into the air before descending to the ground when unpowered. This design allows the vehicle to attain altitudes much greater than current designs. The mass properties and exterior shape of the vehicle were designed to passively reorient the device so that it always rotates to an upright orientation, independent of the landing attitude. The direction of travel is controlled by an internal mass that can rotate around the internal perimeter of the body. This simple mechanism allows the vehicle to tilt in the desired direction before launch and creates a pitching moment once airborne to further accelerate the vehicle.

The work reported here examines flight performance of the hopping rotochute using an experimentally validated dynamic model. Section II develops the hopping rotochute dynamic model, which includes a soft contact model used to estimate the loads and motion during ground contact. Section III describes the test vehicle and the experimental setup used to measure the motion of the hopping rotochute prototype. Section IV describes the methods used to determine the model parameters and compares the experimental and simulated motions to validate the dynamic model. Results of the simulations from the validated dynamic model are presented in Sec. V to demonstrate the basic flight performance of the hopping rotochute. A hop sequence is given that shows the feasibility of using the internal mass as directional control as well as the altitudes and total ranges that a prototype vehicle can achieve.

\section{Hopping Rotochute Dynamic Model}

A schematic of the hopping rotochute system is given in Fig. 1. The hopping rotochute system is modeled with 6 degrees of freedom, including three inertial position components of the total mass center and three Euler orientation angles [7]. In addition to the aerodynamic loads that act on the body, a soft contact model is employed to estimate the applied forces during ground contact. The kinematic and dynamic equations of motion are presented next, as well as the forces and moments that act on the body.

\section{A. Equations of Motion}

The kinematic equations of motion for the vehicle are provided in Eqs. (1) and (2):

$$
\begin{gathered}
\left\{\begin{array}{c}
\dot{x} \\
\dot{y} \\
\dot{z}
\end{array}\right\}=\left[\mathbf{T}_{\mathrm{IB}}\right]\left\{\begin{array}{c}
u \\
v \\
w
\end{array}\right\} \\
\left\{\begin{array}{c}
\dot{\phi} \\
\dot{\theta} \\
\dot{\psi}
\end{array}\right\}=\left[\begin{array}{ccc}
1 & s_{\phi} t_{\theta} & c_{\phi} t_{\theta} \\
0 & c_{\phi} & -s_{\phi} \\
0 & s_{\phi} / c_{\theta} & c_{\phi} / c_{\theta}
\end{array}\right]\left\{\begin{array}{c}
p \\
q \\
r
\end{array}\right\}
\end{gathered}
$$

where the standard shorthand notation is used for trigonometric functions: $\cos (\alpha) \equiv c_{\alpha}, \sin (\alpha) \equiv s_{\alpha}$ and $\tan (\alpha) \equiv t_{\alpha}$. The matrix $\mathbf{T}_{\mathrm{IB}}$ represents the transformation matrix from the body reference frame to an inertial reference frame, which is constructed using the standard aerospace rotation sequence:

$$
\mathbf{T}_{\text {IB }}=\left[\begin{array}{ccc}
c_{\theta} c_{\psi} & s_{\phi} s_{\theta} s_{\psi}-c_{\phi} s_{\psi} & c_{\phi} s_{\theta} c_{\psi}+s_{\phi} s_{\psi} \\
c_{\theta} s_{\psi} & s_{\phi} s_{\theta} s_{\psi}+c_{\theta} c_{\psi} & c_{\phi} s_{\theta} s_{\psi}-s_{\phi} c_{\psi} \\
-s_{\theta} & s_{\phi} c_{\theta} & c_{\phi} c_{\theta}
\end{array}\right]
$$

The dynamic equations are formed by summing forces and moments about the system mass center in the body reference frame and equating the result to the time derivative of linear and angular momentum, respectively. The translational and rotational dynamic equations of motion are expressed as

$$
\begin{gathered}
\left\{\begin{array}{c}
\dot{u} \\
\dot{v} \\
\dot{w}
\end{array}\right\}=\frac{1}{m}\left\{\begin{array}{c}
X \\
Y \\
Z
\end{array}\right\}-\left[\begin{array}{ccc}
0 & -r & q \\
r & 0 & -p \\
-q & p & 0
\end{array}\right]\left\{\begin{array}{c}
u \\
v \\
w
\end{array}\right\} \\
\left\{\begin{array}{c}
\dot{p} \\
\dot{q} \\
\dot{r}
\end{array}\right\}=[I]^{-1}\left(\left\{\begin{array}{c}
L \\
M \\
N
\end{array}\right\}-\left[\begin{array}{ccc}
0 & -r & q \\
r & 0 & -p \\
-q & p & 0
\end{array}\right][I]\left\{\begin{array}{l}
p \\
q \\
r
\end{array}\right\}\right)
\end{gathered}
$$

The mass moment of inertia matrix $I$ is dependent on the location and size of the internal mass. In this study, the internal mass is positioned with respect to the body by a distance $d_{\mathrm{IM}}$ along $\vec{I}_{P}$ from point $P$. Note that the $P$ and $B$ reference frames are related by a rotation $\psi_{\mathrm{IM}}$ about the $\vec{K}_{B}$ axis, as shown in Fig. 1 .

\section{B. Body Forces and Moments}

The applied forces and moments expressed in Eqs. (4) and (5) contain contributions from weight $W$, body BA, rotor RA aerodynamic, and contact $C$ loads. The total forces and moments applied to the vehicle expressed in the body reference frame are given in Eqs. (ㅁ) and (7), respectively:

$$
\begin{gathered}
\left\{\begin{array}{c}
X \\
Y \\
Z
\end{array}\right\}=\left\{\begin{array}{c}
X_{W}+X_{\mathrm{BA}}+X_{\mathrm{RA}}+X_{C} \\
Y_{W}+Y_{\mathrm{BA}}+Y_{\mathrm{RA}}+Y_{C} \\
Z_{W}+Z_{\mathrm{BA}}+Z_{\mathrm{RA}}+Z_{C}
\end{array}\right\} \\
\left\{\begin{array}{c}
L \\
M \\
N
\end{array}\right\}=\left\{\begin{array}{c}
L_{\mathrm{BA}}+L_{\mathrm{RA}}+L_{C} \\
M_{\mathrm{BA}}+M_{\mathrm{RA}}+M_{C} \\
N_{\mathrm{BA}}+N_{\mathrm{RA}}+N_{C}
\end{array}\right\}
\end{gathered}
$$

The weight contribution is given by Eq. ( $\underline{8})$ :

$$
\left\{\begin{array}{c}
X_{W} \\
Y_{W} \\
Z_{W}
\end{array}\right\}=m g\left\{\begin{array}{l}
-s_{\theta} \\
s_{\phi} c_{\theta} \\
c_{\phi} c_{\theta}
\end{array}\right\}
$$

The body aerodynamic force is calculated assuming that only drag acts on the body at the center of pressure CP, as shown in Eq. (9):

$$
\left\{\begin{array}{c}
X_{\mathrm{BA}} \\
Y_{\mathrm{BA}} \\
Z_{\mathrm{BA}}
\end{array}\right\}=-\frac{1}{2} \rho V_{\mathrm{CP}} S C_{D}\left\{\begin{array}{c}
u_{\mathrm{CP}} \\
v_{\mathrm{CP}} \\
w_{\mathrm{CP}}
\end{array}\right\}
$$

Fig. 1 Hopping rotochute schematic. 
where

$$
V_{\mathrm{CP}}=\sqrt{u_{\mathrm{CP}}^{2}+v_{\mathrm{CP}}^{2}+w_{\mathrm{CP}}^{2}}
$$

The body aerodynamic moment about the mass center is calculated using

$$
\left\{\begin{array}{c}
L_{\mathrm{BA}} \\
M_{\mathrm{BA}} \\
N_{\mathrm{BA}}
\end{array}\right\}=\frac{1}{4} \rho V_{\mathrm{CP}} S D^{2}\left\{\begin{array}{c}
C_{l p} p \\
C_{m q} q \\
C_{n r} r
\end{array}\right\}+\left[\mathbf{R}_{\mathrm{CG} \rightarrow \mathrm{CP}]}\left\{\begin{array}{c}
X_{\mathrm{BA}} \\
Y_{\mathrm{BA}} \\
Z_{\mathrm{BA}}
\end{array}\right\}\right.
$$

where $\mathbf{R}_{\mathrm{CG} \rightarrow \mathrm{CP}}$ is the skew-symmetric matrix representation of the position vector from the mass center to the center of pressure of the body along the station line $\left(\mathrm{SL}_{\mathrm{CG} \rightarrow \mathrm{CP}}\right)$, butt line $\left(\mathrm{BL}_{\mathrm{CG} \rightarrow \mathrm{CP}}\right)$, and waterline $\left(\mathrm{WL}_{\mathrm{CG} \rightarrow \mathrm{CP}}\right)$ :

$$
\mathbf{R}_{\mathrm{CG} \rightarrow \mathrm{CP}}=\left[\begin{array}{ccc}
0 & -\mathrm{WL}_{\mathrm{CG} \rightarrow \mathrm{CP}} & \mathrm{BL}_{\mathrm{CG} \rightarrow \mathrm{CP}} \\
\mathrm{WL}_{\mathrm{CG} \rightarrow \mathrm{CP}} & 0 & -\mathrm{SL}_{\mathrm{CG} \rightarrow \mathrm{CP}} \\
-\mathrm{BL}_{\mathrm{CG} \rightarrow \mathrm{CP}} & \mathrm{SL}_{\mathrm{CG} \rightarrow \mathrm{CP}} & 0
\end{array}\right]
$$

The aerodynamic force from the rotor system is given in Eq. (13):

$$
\left\{\begin{array}{c}
X_{\mathrm{RA}} \\
Y_{\mathrm{RA}} \\
Z_{\mathrm{RA}}
\end{array}\right\}=\left[\mathbf{T}_{\mathrm{BR}}\right]\left\{\begin{array}{c}
0 \\
0 \\
-T
\end{array}\right\}
$$

where $T$ is the thrust produced by the rotating rotor system along the $\vec{K}_{R}$ direction. In this development, the rotor frame $R$ is oriented with respect to the body-fixed frame $B$ using the rotation sequence: $\theta_{R}$ about the $\vec{J}_{B}$ axis and then $\phi_{R}$ about the resulting $\vec{I}_{R}$ axis. Using this rotation sequence, the transformation matrix from the rotor frame to the body frame, $\mathbf{T}_{\mathrm{BR}}$, can be constructed and is shown in Eq. (14):

$$
\mathbf{T}_{\mathrm{BR}}=\left[\begin{array}{ccc}
c_{\theta_{R}} & s_{\phi_{R}} s_{\theta_{R}} & c_{\phi_{R}} s_{\theta_{R}} \\
0 & c_{\theta_{R}} & -s_{\phi_{R}} \\
-s_{\theta_{R}} & s_{\phi_{R}} c_{\theta_{R}} & c_{\phi_{R}} c_{\theta_{R}}
\end{array}\right]
$$

The rotor blades of the hopping rotochute do not instantaneously follow the rotor shaft when the body rotates. To capture this phenomenon, two first-order filters are employed to create this lag:

$$
\begin{aligned}
& \dot{\phi}_{F}=\frac{\phi-\phi_{F}}{\tau} \\
& \dot{\theta}_{F}=\frac{\theta-\theta_{F}}{\tau}
\end{aligned}
$$

The resulting filtered states are used to calculate the values of $\phi_{R}$ and $\theta_{R}$, as shown in Eqs. (17) and (18):

$$
\begin{gathered}
\phi_{R}=\phi_{F}-\phi \\
\theta_{R}=\theta_{F}-\theta
\end{gathered}
$$

The aerodynamic moment from the rotor blades is given in Eq. (19):

$$
\left\{\begin{array}{c}
L_{\mathrm{RA}} \\
M_{\mathrm{RA}} \\
N_{\mathrm{RA}}
\end{array}\right\}=\left[\mathbf{R}_{\mathrm{CG} \rightarrow R}\right]\left\{\begin{array}{c}
X_{\mathrm{RA}} \\
Y_{\mathrm{RA}} \\
Z_{\mathrm{RA}}
\end{array}\right\}
$$

The contact forces and moments that act on the body during ground impact are calculated based on a soft contact model originally developed by Goyal et al. [8,9]. The contact model estimates the loads, including Coulomb-like dry friction, by modeling localized nonpermanent material deformation of the contacting surfaces. Vertices are used to approximate the exterior shape of the body (a convex polyhedron), and a horizontal plane (face) represents the ground, as shown in Fig. 2. Contact between each body vertex and the ground face occurs through massless rigid planes called surface elements (SEs). During a contact episode, the SEs are constrained to

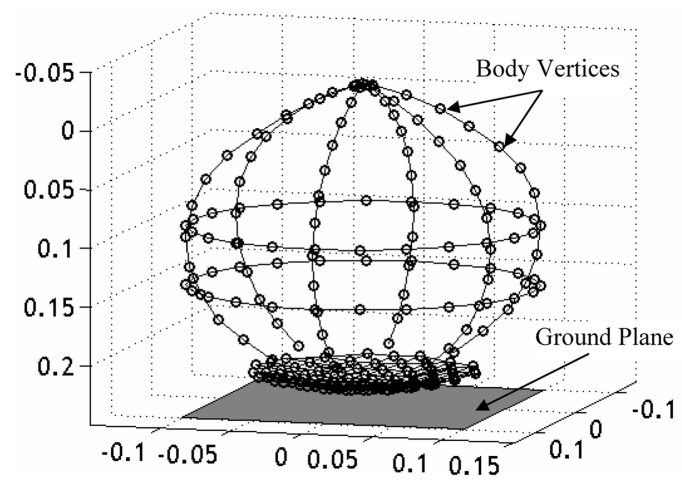

Fig. 2 Example arrangement for the soft contact model body vertices and ground face.

remain parallel to the ground face but can slip against one another. Each body vertex, labeled 1 in Fig. 3 , is connected to an associated surface element through two pairs of massless spring and dampers, as shown in Fig. 3 . The normal spring $k_{1 n}$ and damper $c_{1 n}$ of the body vertices are constrained along the ground-face normal $\vec{n}$, whereas the tangential spring $k_{1 t}$ and damper $c_{1 t}$ always stay in the ground-face plane. The orientation of the tangential elements in this plane is parallel to the direction of slip between the contacting SEs. A similar arrangement of springs and dampers with constants $k_{2 n}, k_{2 t}, c_{2 n}$, and $c_{2 t}$ connects the SEs associated with the ground face (labeled 2 in Fig. 3).

During simulation, a collision detection method determines which body vertices are contacting the ground face and the point of application (point $C$ in Fig. 3). Each contact point has two force components, including a normal component $\vec{F}_{n}$ along $\vec{n}$ and a frictional component $\vec{F}_{t}$ in the tangential plane of contact. The normal and tangential forces from each contact point are given in Eqs. (20) and (21), respectively, where $\vec{s}_{1 n}$ and $\vec{s}_{2 n}$ are the distance vectors of the normal springs and $\vec{s}_{1 t}$ and $\vec{s}_{2 t}$ are the distance vectors of the tangential springs:

$$
\begin{gathered}
\vec{F}_{n}=-k_{1 n} \vec{s}_{1 n}-c_{1 n} \dot{\overrightarrow{\vec{s}}}_{1 n}=-k_{2 n} \vec{s}_{2 n}-c_{2 n} \dot{\overrightarrow{\vec{s}}}_{2 n} \\
\vec{F}_{t}=-k_{1 t} \vec{s}_{1 t}-c_{1 t} \dot{\vec{s}}_{1 t}=-k_{2 t} \vec{s}_{2 t}-c_{2 t} \dot{\vec{s}}_{2 t}
\end{gathered}
$$

The rates of change of the springs are expressed in Eqs. (22) and (23):

$$
\begin{array}{cc}
\dot{\vec{s}}_{1 n}=\vec{u}_{1 n}-\vec{w}_{1 n} & \dot{\vec{s}}_{2 n}=\vec{u}_{2 n}-\vec{w}_{2 n} \\
\dot{\vec{s}}_{1 t}=\vec{u}_{1 t}-\vec{w}_{1 t} & \dot{\vec{s}}_{2 t}=\vec{u}_{2 t}-\vec{w}_{2 t}
\end{array}
$$

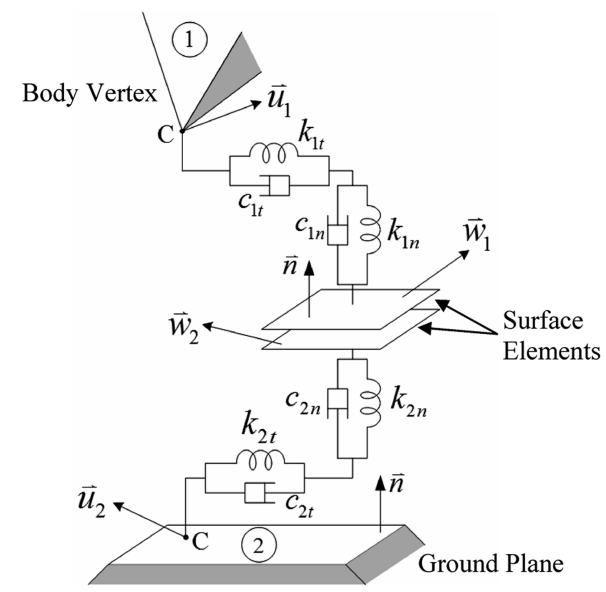

Fig. 3 Spring and damper schematic for the soft contact model. 
where $\vec{u}_{1}$ and $\vec{u}_{2}$ are the absolute velocities of the contact point on the body and on the ground, respectively, and $\vec{w}_{1}$ and $\vec{w}_{2}$ are the absolute velocities of the two SEs. In this development, $\Delta \vec{u}=\vec{u}_{1}-\vec{u}_{2}, \Delta \vec{w}=$ $\vec{w}_{1}-\vec{w}_{2}$, and $\Delta \vec{w}_{n}=0$, because no relative motion is allowed between the SEs along $\vec{n}$. Substituting these equations into Eqs. (22) and (23) yields

$$
\dot{\vec{s}}_{1 n}-\dot{\vec{s}}_{2 n}=\Delta \vec{u}_{n} \quad \dot{\vec{s}}_{1 t}-\dot{\vec{s}}_{2 t}=\Delta \vec{u}_{t}-\Delta \vec{w}_{t}
$$

The contact force of a contact point in the normal direction and the tangential plane is formed by manipulating Eqs. (20), (21), and (24) to yield

$$
\begin{gathered}
\vec{F}_{n}=-\vec{b}_{n} \\
\vec{F}_{t}=-\vec{b}_{t}+c^{*} \Delta \vec{w}_{t}
\end{gathered}
$$

where

$$
\begin{gathered}
c^{*}=\frac{c_{1 t} c_{2 t}}{c_{1 t}+c_{2 t}} \\
\vec{b}_{n}=\frac{1}{c_{1 n}+c_{2 n}}\left(c_{2 n} k_{1 n} \vec{s}_{1 n}-c_{1 n} k_{2 n} \vec{s}_{2 n}+c_{1 n} c_{2 n} \Delta \vec{u}_{n}\right) \\
\vec{b}_{t}=\frac{1}{c_{1 t}+c_{2 t}}\left(c_{2 t} k_{1 t} \vec{s}_{1 t}-c_{1 t} k_{2 t} \vec{s}_{2 t}+c_{1 t} c_{2 t} \Delta \vec{u}_{t}\right)
\end{gathered}
$$

With the coefficient of friction between the two surfaces denoted as $\mu$, and with $\lambda$ as a nonnegative real variable, the friction law can be expressed as

$$
\left|\vec{F}_{t}\right| \leq \mu\left|\vec{F}_{n}\right| \quad \Delta \vec{w}_{t}=-\lambda \vec{F}_{t} \quad \lambda \geq 0
$$

Substituting Eqs. (25) and (26) into Eq. (무) results in

$$
\frac{\left|\vec{b}_{t}\right|}{\left(1+\lambda c^{*}\right)} \leq \mu\left|\vec{b}_{n}\right|
$$

A state of stick exists between the two surface elements when $\left|\vec{b}_{t}\right| \leq \mu\left|\vec{b}_{n}\right|$. While in a state of stick, $\lambda=0$ and $\Delta \vec{w}_{t}=0$, which from Eq. (26) implies that $\vec{F}_{t}=-\vec{b}_{t}$. If, on the other hand, $\left|\vec{b}_{t}\right|>$ $\mu\left|\vec{b}_{n}\right|$, the value of $\lambda$ that makes Eq. (31) an equality is

$$
\lambda=\frac{\left|\vec{b}_{t}\right|-\mu\left|\vec{b}_{n}\right|}{c^{*} \mu\left|\vec{b}_{n}\right|}
$$

In this case, $\Delta \vec{w}_{t}$ and $\vec{F}_{t}$ can be calculated using Eqs. (26) and (무) using the value of $\lambda$ calculated from Eq. (32):

$$
\begin{aligned}
\Delta \vec{w}_{t} & =\frac{\lambda \vec{b}_{t}}{\left(1+\lambda c^{*}\right)} \\
\vec{F}_{t} & =\frac{-\vec{b}_{t}}{\left(1+\lambda c^{*}\right)}
\end{aligned}
$$

The total contact force applied to the body in the body reference frame is calculated by summing the forces produced at each contact point, as given in Eq. (35):

$$
\left\{\begin{array}{l}
X_{C} \\
Y_{C} \\
Z_{C}
\end{array}\right\}=\left[\mathbf{T}_{\mathrm{BI}}\right] \sum_{C=1}^{\mathrm{NC}}\left(\left\{\begin{array}{l}
F_{n_{x}}+F_{t_{x}} \\
F_{n_{y}}+F_{t_{y}} \\
F_{n_{s}}+F_{t_{s}}
\end{array}\right\}\right)
$$

where NC is the number of contact points. The moment about the mass center produced by the contact is calculated by crossing the distance vector from the mass center to each contact point with the force exerted at that contact point, as shown in Eq. (36):

$$
\left\{\begin{array}{c}
L_{C} \\
M_{C} \\
N_{C}
\end{array}\right\}=\sum_{C=1}^{\mathrm{NC}}\left(\left[\mathbf{R}_{\mathrm{CG} \rightarrow C}\right]\left[\mathbf{T}_{B I}\right]\left\{\begin{array}{l}
F_{n_{x}}+F_{t_{x}} \\
F_{n_{y}}+F_{t_{y}} \\
F_{n_{s}}+F_{t_{s}}
\end{array}\right\}\right)
$$

The state of the springs in the contact mechanism are tracked with the following differential equations, obtained from Eqs. (20), (21), and (24):

$$
\begin{gathered}
\dot{\vec{s}}_{1 n}=\frac{c_{2 n}}{c_{1 n}+c_{2 n}} \Delta \vec{u}_{n}-\frac{1}{c_{1 n}+c_{2 n}}\left(k_{1 n} \vec{s}_{1 n}+k_{2 n} \vec{s}_{2 n}\right) \\
\dot{\vec{s}}_{2 n}=\frac{-c_{1 n}}{c_{1 n}+c_{2 n}} \Delta \vec{u}_{n}-\frac{1}{c_{1 n}+c_{2 n}}\left(k_{1 n} \vec{s}_{1 n}+k_{2 n} \vec{s}_{2 n}\right) \\
\dot{\vec{s}}_{1 t}=\frac{c_{2 t}}{c_{1 t}+c_{2 t}}\left(\Delta \vec{u}_{t}-\Delta \vec{w}_{t}\right)-\frac{1}{c_{1 t}+c_{2 t}}\left(k_{1 t} \vec{s}_{1 t}+k_{2 t} \vec{s}_{2 t}\right) \\
\dot{\vec{s}}_{2 t}=\frac{-c_{1 t}}{c_{1 t}+c_{2 t}}\left(\Delta \vec{u}_{t}-\Delta \vec{w}_{t}\right)-\frac{1}{c_{1 t}+c_{2 t}}\left(k_{1 t} \vec{s}_{1 t}+k_{2 t} \vec{s}_{2 t}\right)
\end{gathered}
$$

During this study, 223 vertices were used to approximate the exterior shape of the hopping rotochute body. Based on this, the simulation tracks 2690 states with the dynamic equations given in Eqs. (1), (2), (4), (5), (15), (16), and (37-40). Of course, during the simulation not all of the body vertices are in contact with the ground. A collision detection method determines which of the body vertices are in ground contact and these contact points are used to calculate the contact forces and moments using Eqs. (35) and (36), respectively.

\section{Vehicle and Motion Measurement System Description}

\section{A. Test Vehicle}

The hopping rotochute prototype used in this study is shown in Fig. 4 and is remotely controlled using a Futaba radio and a microreceiver. The vehicle consists of an expanded polypropylene foam core, a polyurethane foam cushion, a carbon fiber cage, an internal mass, associated electronics, and a transmission and rotor system that were taken from an Air Hogs Reflex Micro Helicopter. Both sets of the rotors are driven by a single small brushed dc electric motor that is controlled by an electronic speed controller. The rotor blades have a radius of $10.6 \mathrm{~cm}$ and a mean aerodynamic chord of $2 \mathrm{~cm}$. The internal mass is able to rotate $\pm 180 \mathrm{deg}$ around the internal perimeter of the body by employing a micro servo and gear train. The carbon fiber frame houses the rotating components and was shaped specifically to upright the vehicle when on the ground. The prototype has a height of $25.4 \mathrm{~cm}$ and a maximum horizontal cage diameter of $24.8 \mathrm{~cm}$. The mass properties of the hopping rotochute prototype including a $250 \mathrm{mAh}$ lithium polymer battery pack, six markers, and a $6 \mathrm{~g}$ internal mass located $6 \mathrm{~cm}$ along $-\vec{I}_{B}$ from point $P\left(d_{\mathrm{IM}}=\right.$ $6 \mathrm{~cm}, \psi_{\mathrm{IM}}=180 \mathrm{deg}$ ) are outlined in Table 1 . Note that although the internal mass is able to rotate around the internal perimeter, the mass properties given in Table 1 are associated with a fixed internal mass used in the validation process.

\section{B. Instrumentation}

The motion of the hopping rotochute was experimentally obtained using a 3-D optical position analysis system from Vicon Motion Systems. The 12-camera motion capture system is housed in the Indoor Flight Facility (IFF) at Georgia Institute of Technology, 


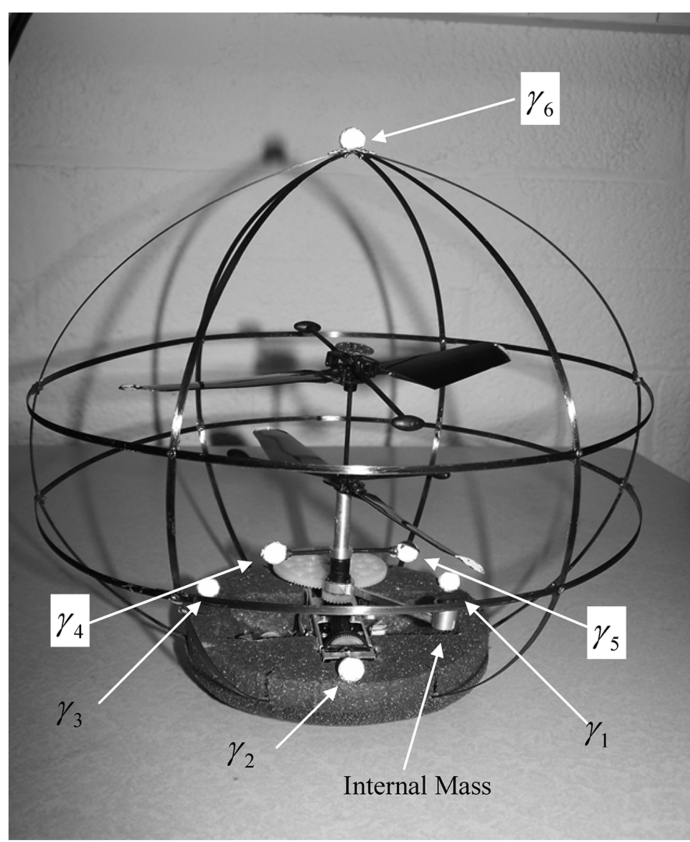

Fig. 4 Hopping rotochute prototype with Vicon markers.

which is $7.6 \mathrm{~m}$ wide, $8.5 \mathrm{~m}$ long, and $3.6 \mathrm{~m}$ high. As shown in Fig. 5, the cameras are strategically arranged around the room so that the capture volume can encompass the entire room. Two cameras are located at each corner of the room and a single camera is situated in the middle of each of the four walls. The cameras emit and collect visible infrared light at a frequency up to 2000 frames/s from spherical retroreflective markers that are rigidly attached to the hopping rotochute. Optical correlation techniques are employed to determine the three-dimensional position of the center of each marker to within $1 \mathrm{~mm}$ accuracy in real time. The time-stamped data are stored in a text file and are subsequently used to determine the position, orientation, velocity, and angular velocity of the body.

\section{Data Processing}

As shown in Fig. 4, six 9-mm-diam markers were used during experimental testing of the hopping rotochute. Three of the markers were rigidly attached to the base, two were secured to the lower rotor shaft, and the other was placed on top of the cage. To generate the mass center position and orientation of the body, marker position data are processed to generate the transformation matrix from the body

Table 1 Hopping rotochute properties

\begin{tabular}{lcc}
\hline \hline Parameter & Value & Units \\
\hline$m$ & 91.1 & $\mathrm{~g}$ \\
$\mathrm{SL}_{F \rightarrow \mathrm{CG}}, \mathrm{BL}_{F \rightarrow \mathrm{CG}}, \mathrm{WL}_{F \rightarrow \mathrm{CG}}$ & $-0.33,0.0,20.0$ & $\mathrm{~cm}$ \\
$I_{X X}, I_{Y Y}, I_{Z Z}$ & $4011,4292,1013$ & $\mathrm{~g} \cdot \mathrm{cm}^{2}$ \\
$I_{X Y}, I_{X Z}, I_{Y Z}$ & $0,70,0$ & $\mathrm{~g} \cdot \mathrm{cm}^{2}$ \\
$k_{1 n}, k_{1 t}$ & 212,212 & $\mathrm{~N} / \mathrm{m}$ \\
$k_{2 n}, k_{2 t}$ & 120,120 & $\mathrm{~N} / \mathrm{m}$ \\
$c_{1 n}, c_{1 t}$ & $0.5,0.5$ & $\mathrm{~N} \cdot \mathrm{s} / \mathrm{m}$ \\
$c_{2 n}, c_{2 t}$ & $0.5,0.5$ & $\mathrm{~N} \cdot \mathrm{s} / \mathrm{m}$ \\
$\mu$ & 3.0 & - \\
$\mathrm{SL}_{F \rightarrow P}, \mathrm{BL}_{F \rightarrow P}, \mathrm{WL}_{F \rightarrow P}$ & $0.0,0.0,22.0$ & $\mathrm{~cm}$ \\
$\mathrm{SL}_{F \rightarrow R}, \mathrm{BL}_{F \rightarrow R}, \mathrm{WL}_{F \rightarrow R}$ & $0.0,0.0,12.7$ & $\mathrm{~cm}$ \\
$\mathrm{SL}_{F \rightarrow \mathrm{CP}}, \mathrm{BL}_{F \rightarrow \mathrm{CP}}$ & $0.0,0.0$ & $\mathrm{~cm}$ \\
$C_{D}($ powered flight $)$ & 1.0 & - \\
$\mathrm{WL}_{F \rightarrow \mathrm{CP}}($ powered flight$)$ & 16.0 & $\mathrm{~cm}$ \\
$C_{D}($ unpowered flight) & 0.6 & - \\
$\mathrm{WL}_{F \rightarrow \mathrm{CP}}$ (unpowered flight) & 19.0 & $\mathrm{~cm}$ \\
$C_{l p}, C_{m q}, C_{n r}$ & -0.3 & - \\
$D$ & 25.4 & $\mathrm{~cm}$ \\
$S$ & 506.7 & $\mathrm{~cm}$ \\
$\tau$ & 0.17 & $\mathrm{~s}$ \\
\hline \hline
\end{tabular}

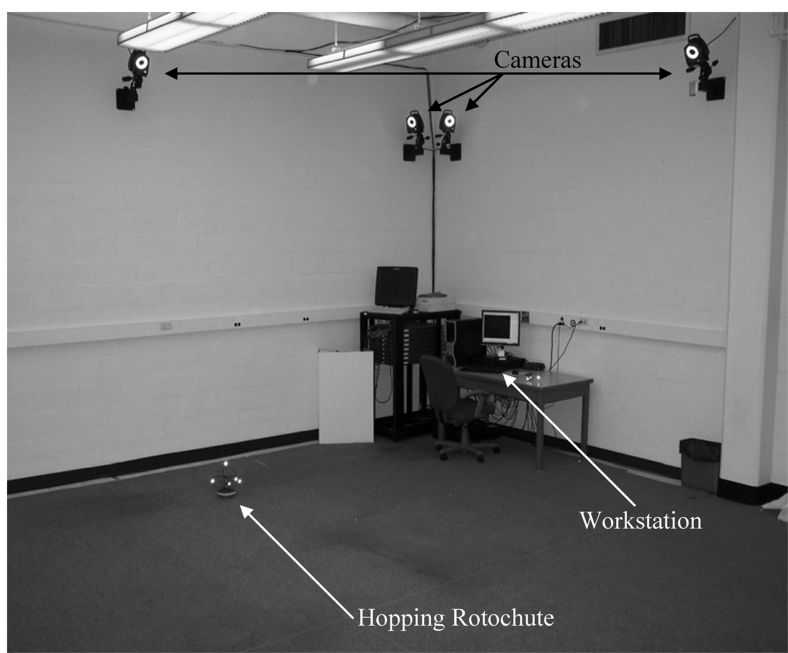

Fig. 5 Indoor flight facility with Vicon motion capture system.

reference frame to an inertial frame. To illustrate this process, consider the markers labeled $\gamma_{1}, \gamma_{2}$, and $\gamma_{3}$ attached to the base of the hopping rotochute shown in Fig. 4 . The body reference frame unit vectors are defined such that the $\vec{J}_{B}$ axis points laterally from marker $\gamma_{1}$ to $\gamma_{3}$, the $\vec{K}_{B}$ axis points vertically downward, and the $\vec{I}_{B}$ axis points forward (normal to the plane created by the $\vec{J}_{B}$ and $\vec{K}_{B}$ axes). Mathematically, these three markers are used to define the body reference frame using Eqs. (1ㅡㄴㅡ):

$$
\begin{gathered}
\vec{J}_{B}=\frac{\vec{r}_{O \rightarrow \gamma_{3}}-\vec{r}_{O \rightarrow \gamma_{1}}}{\left\|\vec{r}_{O \rightarrow \gamma_{3}}-\vec{r}_{O \rightarrow \gamma_{1}}\right\|}=j_{B x} \vec{I}_{I}+j_{B y} \vec{J}_{I}+j_{B z} \vec{K}_{I} \\
\vec{K}_{B}=\frac{\left(\vec{r}_{O \rightarrow \gamma_{2}}-\vec{r}_{O \rightarrow \gamma_{1}}\right) \times \vec{J}_{B}}{\left\|\left(\vec{r}_{O \rightarrow \gamma_{2}}-\vec{r}_{O \rightarrow \gamma_{1}}\right) \times \vec{J}_{B}\right\|}=k_{B x} \vec{I}_{I}+k_{B y} \vec{J}_{I}+k_{B z} \vec{K}_{I} \\
\vec{I}_{B}=\frac{\vec{J}_{B} \vec{K}_{B}}{\left\|\vec{J}_{B} \times \vec{K}_{B}\right\|}=i_{B x} \vec{I}_{I}+i_{B y} \vec{J}_{I}+i_{B z} \vec{K}_{I}
\end{gathered}
$$

Note that the body reference frame and the inertial reference frame unit vectors are related by Eq. (44):

$$
\left\{\begin{array}{l}
\vec{I}_{B} \\
\vec{J}_{B} \\
\vec{K}_{B}
\end{array}\right\}=\left[\mathbf{T}_{\mathrm{BI}}\right]\left\{\begin{array}{l}
\vec{I}_{I} \\
\vec{J}_{I} \\
\vec{K}_{I}
\end{array}\right\}
$$

Using this relationship and equating the components resolved in the inertial frame yields

$$
\left[\begin{array}{lll}
i_{B x} & j_{B x} & k_{B x} \\
i_{B y} & j_{B y} & k_{B y} \\
i_{B z} & j_{B z} & k_{B z}
\end{array}\right]=\left[\mathbf{T}_{\mathrm{IB}}\right]
$$

The Euler orientation angles of the body are obtained by comparing the functional form of the transformation matrix in Eq. (3) with the measured transformation matrix in Eq. (45), yielding

$$
\begin{gathered}
\theta=-\sin ^{-1}\left(i_{B z}\right) \\
\phi=\tan ^{-1}\left(\frac{j_{B z}}{k_{B z}}\right)
\end{gathered}
$$




$$
\psi=\tan ^{-1}\left(\frac{i_{B y}}{i_{B x}}\right)
$$

The position and orientation data are numerically differentiated using a second-order finite difference method, and a running average with a 10-sample window size is implemented to reduce noise amplification. The results of this procedure are substituted into Eqs. (1) and (2) to obtain the components of the linear and angular velocities in the body reference frame. A similar approach is taken to calculate the rotor speed using the markers labeled $\gamma_{4}, \gamma_{5}$, and $\gamma_{6}$ in Fig. 4 .

\section{Dynamic Model Parameter Estimation and Validation}

To validate the dynamic model of the hopping rotochute, the prototype was tested in two different scenarios and the results were compared with that estimated by the simulation. For the first scenario, the spring and damper constants were experimentally determined and a drop test was performed to determine the validity of the contact model. During the second scenario, the captured flight dynamics of the prototype were compared with the simulation results, which incorporate experimentally acquired aerodynamic characteristics. The details of these tests are discussed subsequently. Note that during these validation tests the internal mass orientation was fixed, and the mass properties of the tested prototype are outlined in Table 1 .

\section{A. Soft Contact Model Parameter Estimation and Validation}

The soft contact model requires the values of the spring constant $k$ and the damping coefficient $c$ of both the polyurethane foam cushion material on the bottom of the hopping rotochute and the carpet covering the ground of the IFF. In addition, the coefficient of friction $\mu$ is needed between these two materials. Three different procedures were used for the determination of these material characteristics. To find the spring constants, material samples were placed on a scale and deflected a known amount while the exerted force was recorded. Using Hooke's law, $F=-k x$, the spring constants were determined to be approximately $12,120 \mathrm{~N} / \mathrm{m}$ for the polyurethane foam and $6800 \mathrm{~N} / \mathrm{m}$ for the carpet. These spring constants assume that only one vertex exists for the sample material area of $20.3 \mathrm{~cm}^{2}$. Because the contact model consists of many vertices that are approximately equal distance from each other, there are more vertices per area. To incorporate this into the model, an equivalent spring constant was calculated based on the vertex spacing. During this study, a vertex spacing of $6.9 \mathrm{~mm}$ was used, which gives an equivalent spring constant of $212 \mathrm{~N} / \mathrm{m}$ for the polyurethane foam and $120 \mathrm{~N} / \mathrm{m}$ for the carpet, as shown in Table 1 .

To obtain the damping coefficients of each material, a drop-test stand was fabricated. The drop-test stand allowed a material sample to drop onto another material sample while constraining the motion to 1 degree of freedom. The motion was recorded by the motion capture system and subsequently compared with simulation results using the same initial conditions and mass properties as well as the spring constants from the test previously described. The damping coefficients were adjusted until the experimental trajectory matched the simulation results. The damping coefficient along the normal and tangential directions associated with a vertex spacing of $6.9 \mathrm{~mm}$ was determined to be approximately $0.5 \mathrm{~N} \cdot \mathrm{s} / \mathrm{m}$ for both the polyurethane and the carpet, as outlined in Table 1.

The coefficient of friction was obtained by placing a weight of known mass on a polyurethane foam sample and moving it at a constant speed across the carpet while measuring the force. Using the friction law, $F=\mu N$, the coefficient of friction between the polyurethane foam and the carpet was calculated to be about 3.0.

With the contact model parameters determined, the motion of the hopping rotochute was captured and compared with the dynamic model simulation results to determine the validity of the soft contact model. The prototype was dropped from a height of $1.2 \mathrm{~m}$ without applying power to the rotor system and the motion was recorded

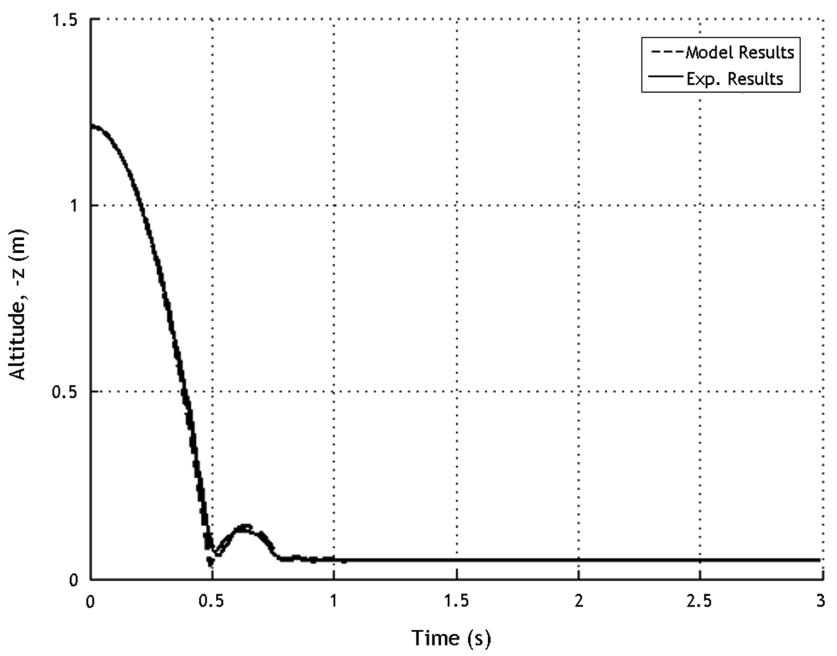

Fig. 6 Drop-test altitude versus time.

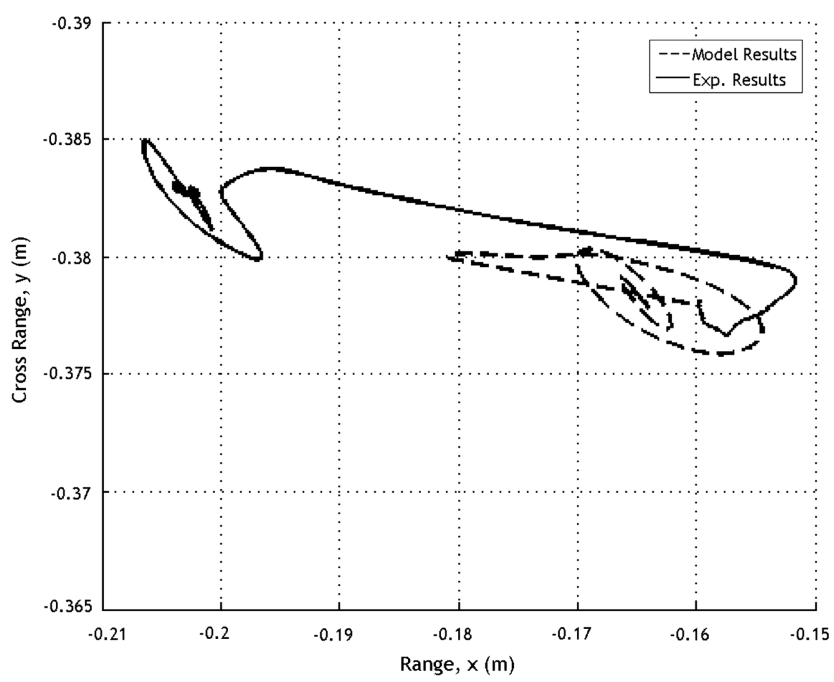

Fig. 7 Drop-test cross range versus range.

using the motion capture system. The same drop was simulated with the experimentally tuned dynamic model using the same initial conditions and the results are shown in Figs. 6-9, in which the solid line and dashed line represent the captured and simulated data, respectively. As shown in Fig. $\underline{6}$, the prototype hopping rotochute

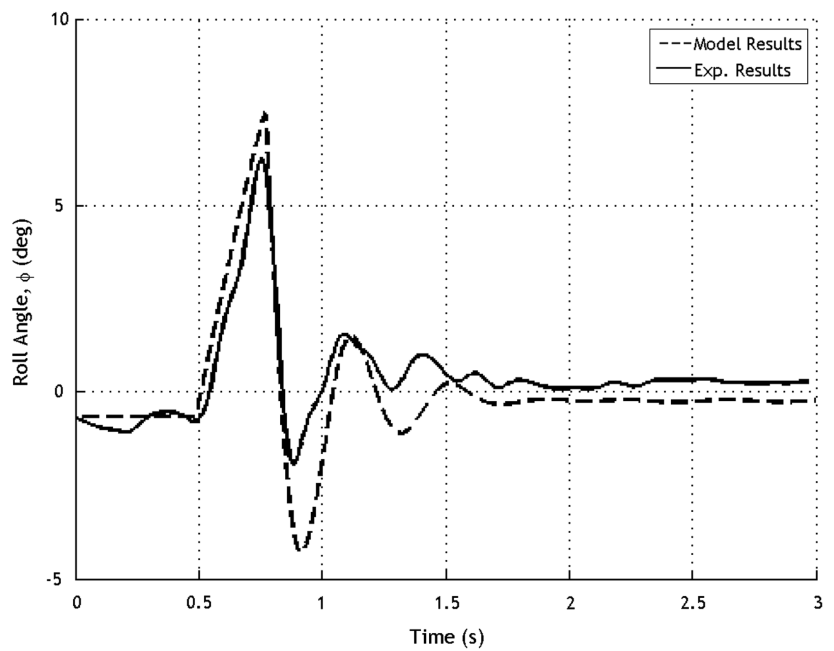

Fig. 8 Drop-test roll angle versus time. 


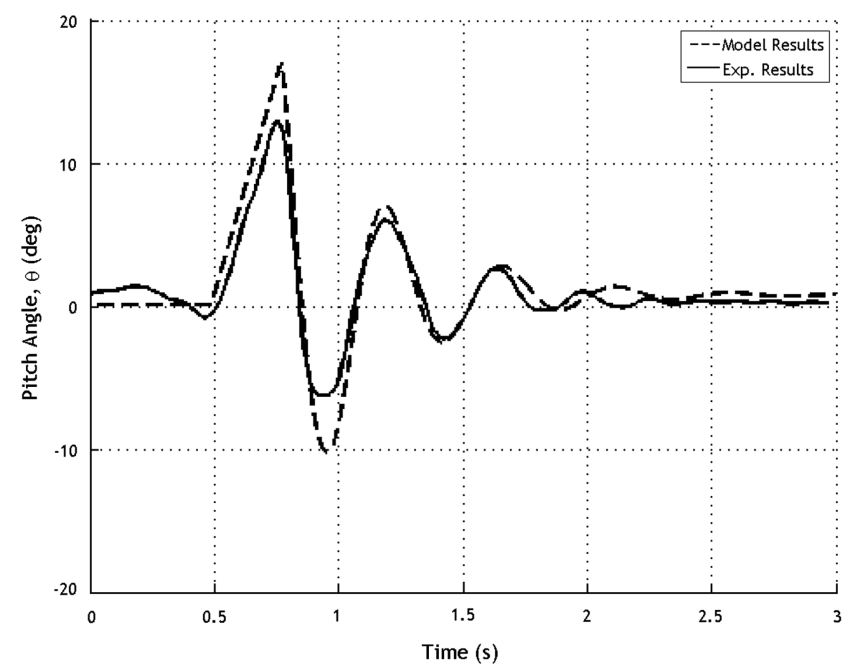

Fig. 9 Drop-test pitch angle versus time.

bounces to a height of about $13 \mathrm{~cm}$ after ground impact, whereas the simulation predicts a height of $14 \mathrm{~cm}$. Figure 7 demonstrates that the general behavior of the range and cross range motion is produced by the simulation, although the simulated drop does not travel as far in the horizontal plane. Figures $\underline{8}$ and $\underline{9}$ present the roll and pitch angles as a function of time. As shown, the initial impact rolls the prototype up to about $6.2 \mathrm{deg}$ while pitching it to $13 \mathrm{deg}$. The simulation, on the other hand, predicts values of 7.5 and $17 \mathrm{deg}$, respectively. Figures $\underline{8}$ and 9 also demonstrate that the frequency and damping ratio of the simulated rolling and pitching oscillations agree with the captured motion when in ground contact.

\section{B. Flight Dynamic Model Parameter Estimation and Validation}

The hopping rotochute dynamic model includes rotor and body aerodynamic loads that must be characterized. The aerodynamic properties of the rotor system were obtained via a rotor test stand. Using this setup, the thrust produced by the rotor system and the current and voltage going through and across the motor were measured at several rotor heights above the ground $(10.2-38.1 \mathrm{~cm})$ and rotor speeds (up to $2100 \mathrm{rpm}$ ). Curves were fitted to the resulting data, extrapolated to $4000 \mathrm{rpm}$, and used to calculate the power. The rotor lag time constant was experimentally obtained by videotaping the powered rotor system, and the prototype was pitched quickly by 90 deg about a point on the base. The pitch angle of the body and the angle of the tip path plane were measured throughout the timestamped video footage and these data were compared with the results of a first-order filter to determine the time constant. The rotor lag time constant was found to be approximately $0.17 \mathrm{~s}$. The body aerodynamic coefficient and center of pressure were obtained by analyzing data collected from the motion capture system and performing a force and moment balance to determine these unknowns. During powered flight, the drag coefficient was determined to be about 1.0 and the center of pressure was calculated to be approximately $16 \mathrm{~cm}$ along $\vec{K}_{B}$ from point $F$, which is right below the lower rotor blades. Note that this aerodynamic force accounts for the additional drag on the body while the rotors are spinning, because download is already captured by the results of the rotor test stand experiment. The body aerodynamic coefficient and center of pressure during unpowered

flight were determined to be 0.6 and $19 \mathrm{~cm}$, respectively, along $\vec{K}_{B}$ from point $F$. The aerodynamic damping moment coefficients were experimentally determined to be approximately -0.3 . The aerodynamic properties of the hopping rotochute are tabulated in Table 1 .

To validate the flight dynamics part of the hopping rotochute model, the motion capture system was employed to record an example hop and the results were compared with simulation results that incorporated the same initial conditions, the model parameters described previously and in Table $\underline{1}$, and the mass properties outlined

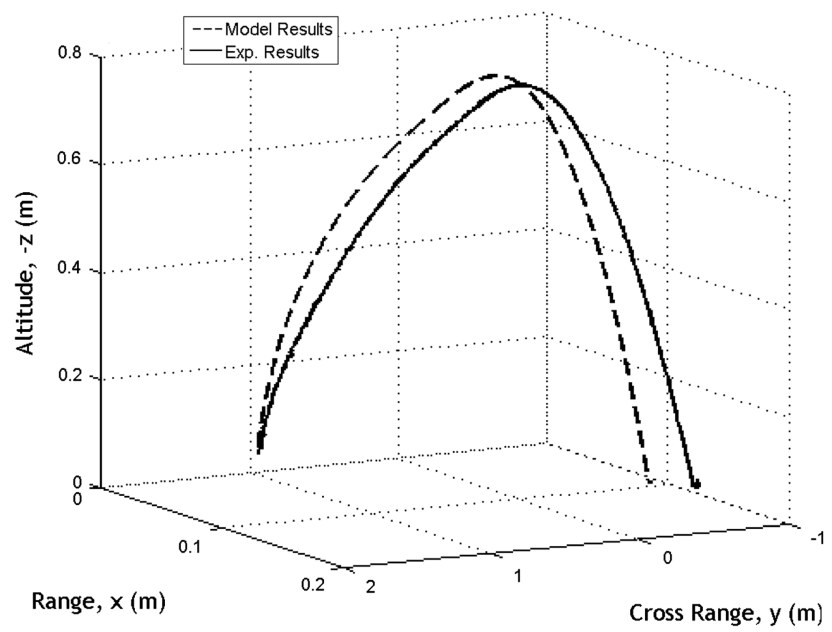

Fig. 10 Flight-test altitude versus cross range versus range.

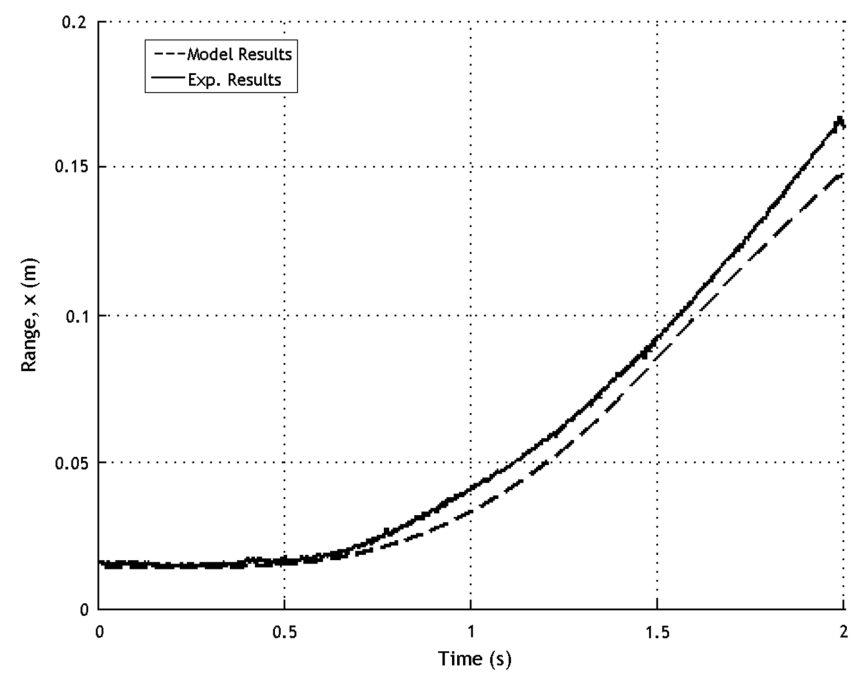

Fig. 11 Flight-test range versus time.

in Table 1. The time histories of the example hop recorded by the motion capture system as well as the results from the simulation are shown in Figs. 10-19. In these charts, the solid lines represent the data from the motion capture system and the dashed lines represent the simulated results. As shown in Figs. $10-13$, the mass center

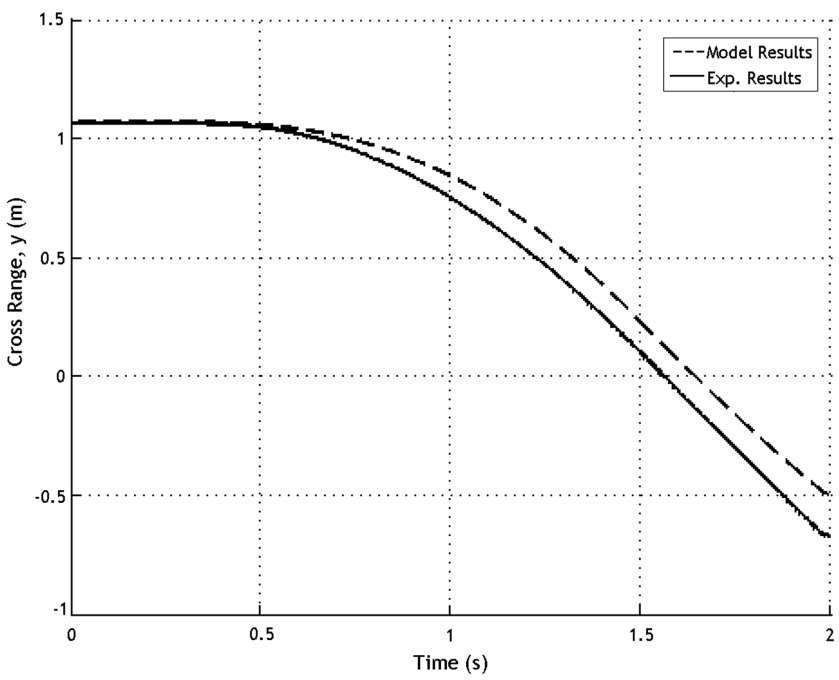

Fig. 12 Flight-test cross range versus time. 


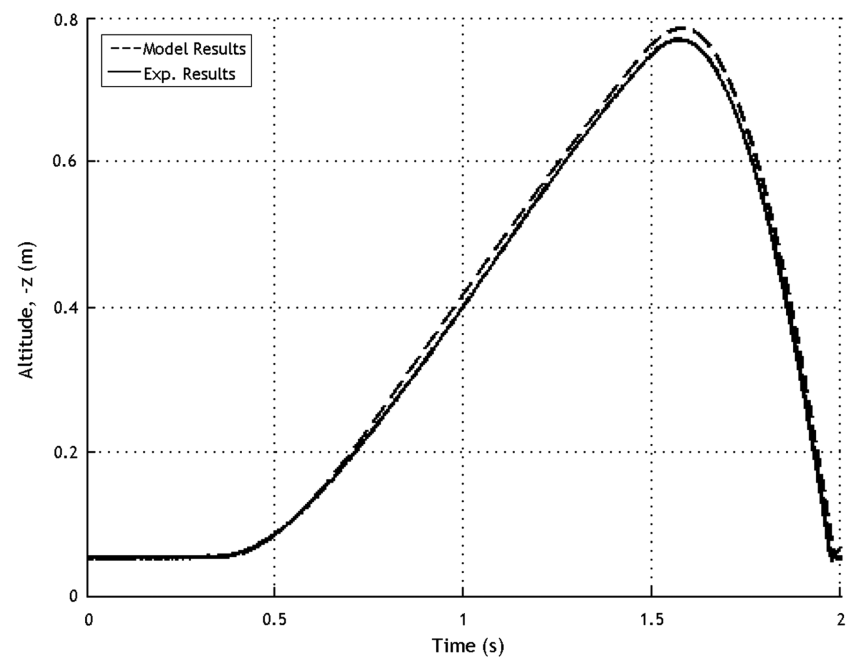

Fig. 13 Flight-test altitude versus time.

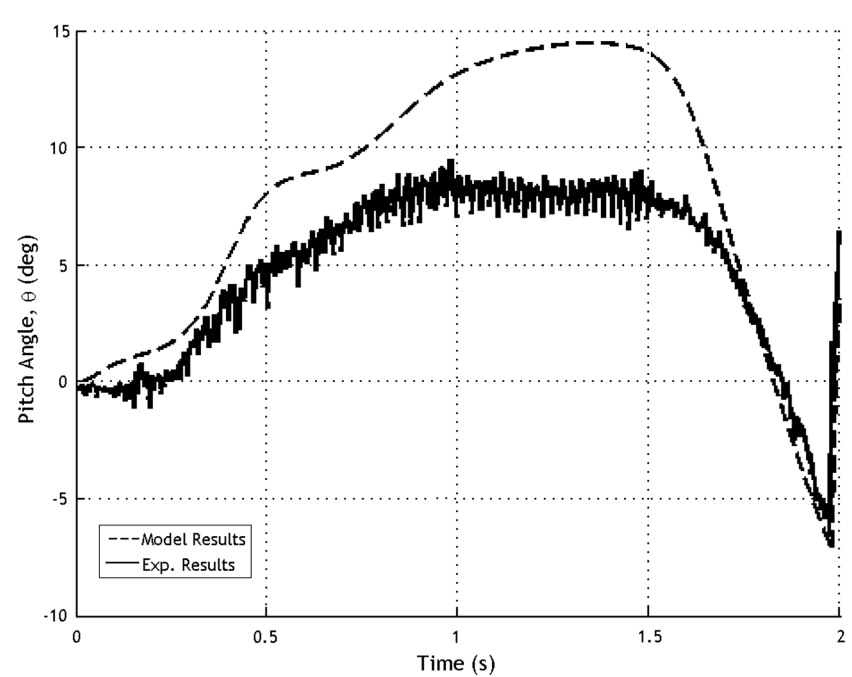

Fig. 14 Flight-test pitch angle versus time.

position versus time associated with the captured and simulated results correlate well. For this particular hop, the hopping rotochute reached a maximum altitude of about $77 \mathrm{~cm}$ at $1.6 \mathrm{~s}$ while achieving a total range of $1.73 \mathrm{~m}$ associated with the captured data and $1.57 \mathrm{~m}$ for

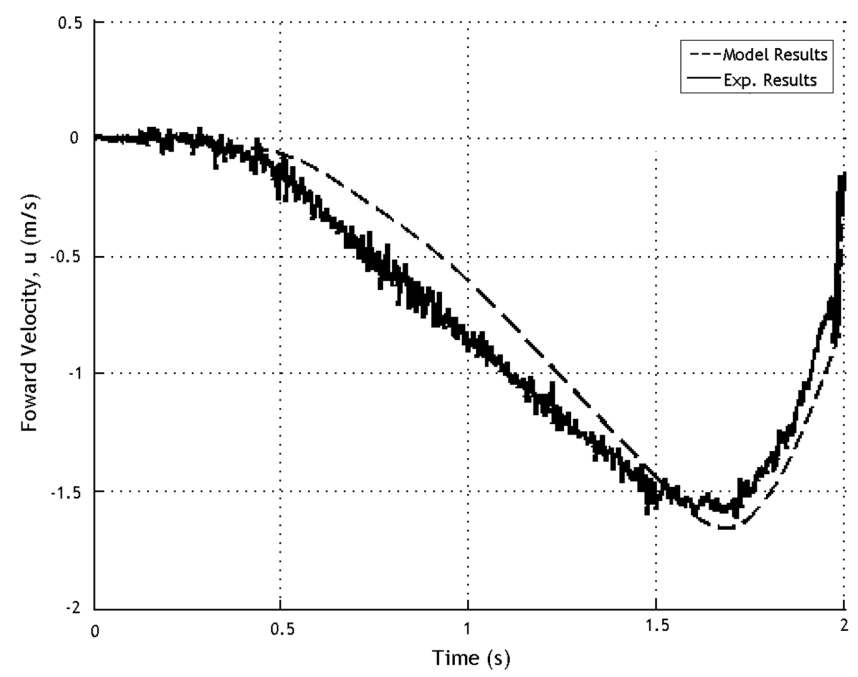

Fig. 15 Flight-test forward velocity versus time.

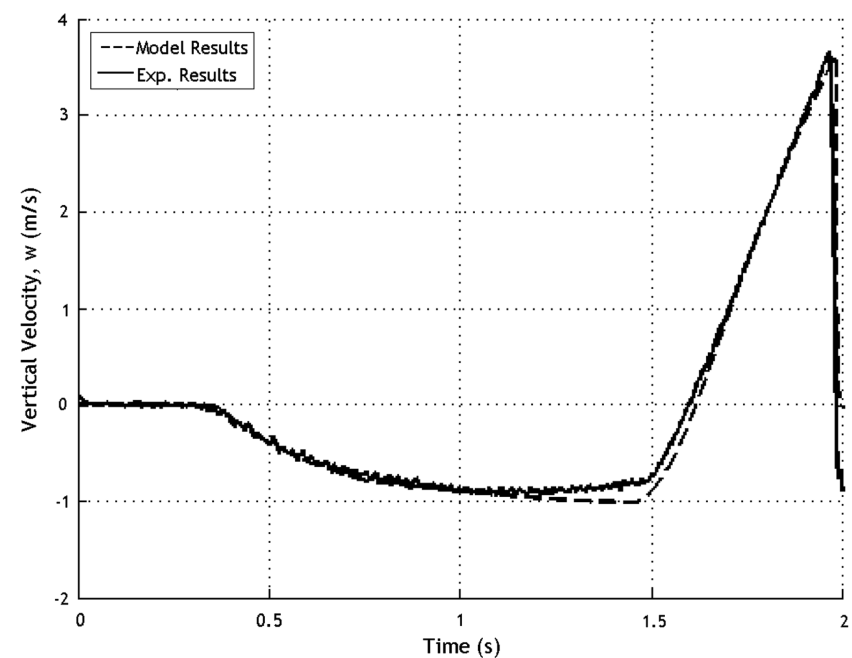

Fig. 16 Flight-test vertical velocity versus time.

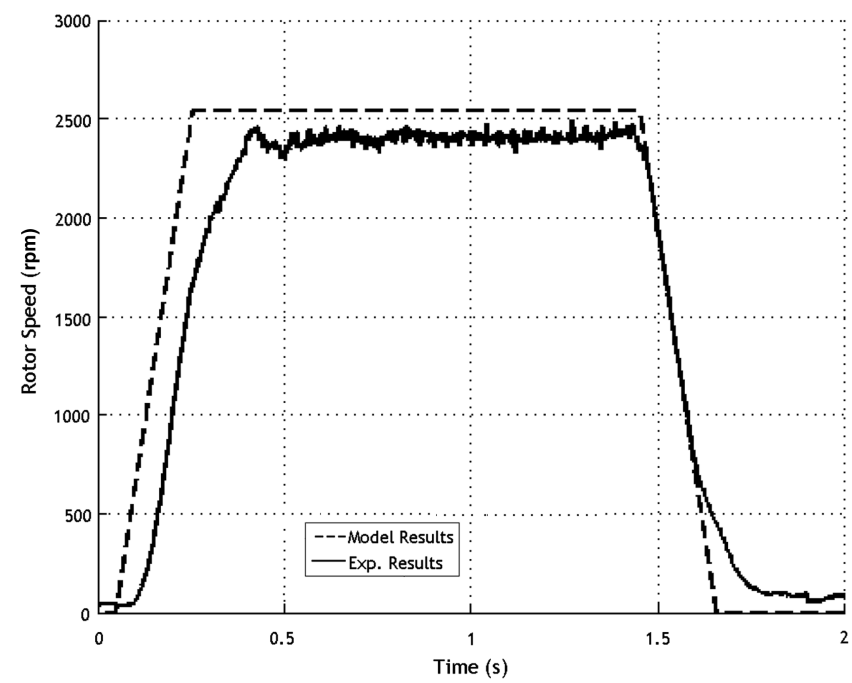

Fig. 17 Flight-test rotor speed versus time.

the simulated results. The pitch angle versus time is given in Fig. 14. The captured pitch angle increases to a value of $8.5 \mathrm{deg}$, whereas the simulated pitch angle reaches a maximum of $14.5 \mathrm{deg}$ before rapidly decreasing after $1.5 \mathrm{~s}$ while the vehicle is unpowered. Although the

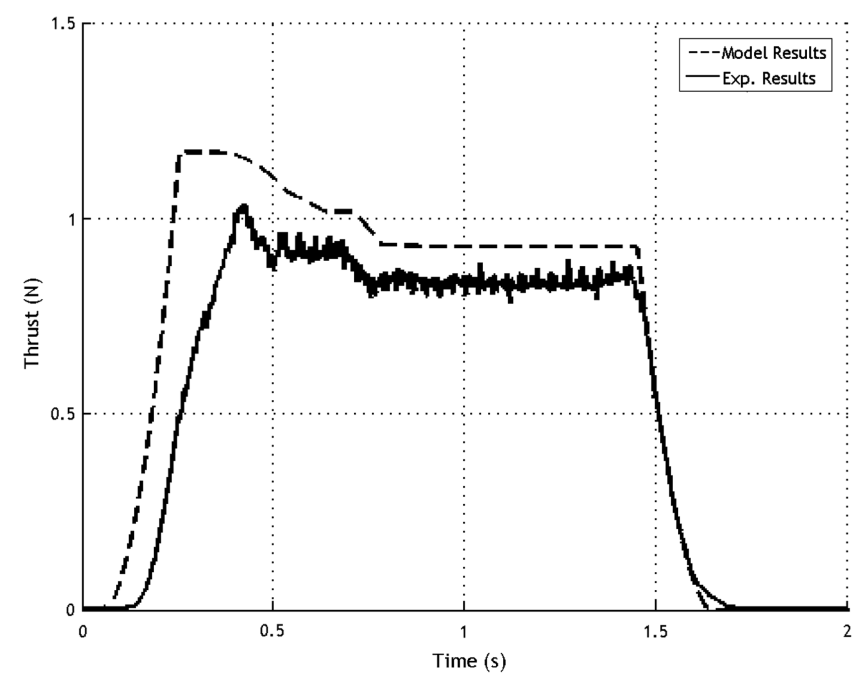

Fig. 18 Flight-test thrust versus time. 


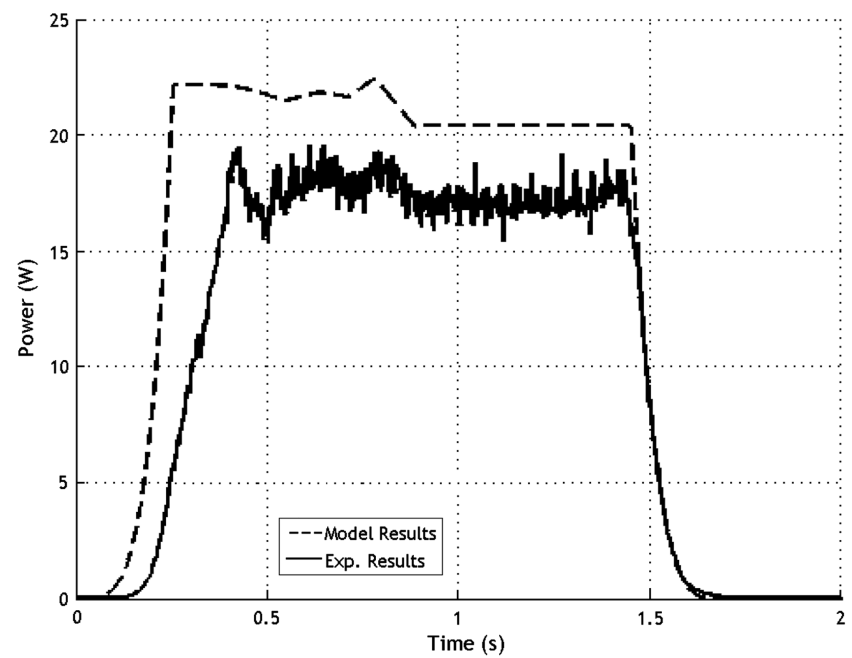

Fig. 19 Flight-test power versus time.

recorded pitch angle is consistently lower than that resulting from the simulation, the general trend is captured. Figures 15 and 16 compare forward and vertical velocities of the captured and simulated motion. The forward velocity decreases almost linearly to a value of $-1.6 \mathrm{~m} / \mathrm{s}$ associated with the captured data and -1.66 for the simulated result while the vehicle is powered and subsequently increases once the power is ceased. The vertical velocity exponentially decreases and plateaus at approximately $-0.9 \mathrm{~m} / \mathrm{s}$ before rapidly increasing when unpowered until ground impact occurs. Figure 17 presents the rotor speed profiles associated with the captured and simulated data. The captured rotor speed is conservative and has a time lag when compared with the simulated rotor speed due to marker exclusions and the calculation routine. The simulated rotor speed ramps up to $2540 \mathrm{rpm}$, whereas the captured rotor speed achieves a maximum average value $2420 \mathrm{rpm}$. Because the rotor thrust and power are based on the rotor speed profile, the same type of amplitude and time-lag trends occur for these time histories, as presented in Figs. 18 and 19. As shown, the thrust and power initially peak due to the high rotor speed and in ground effect and then level off before returning back to zero. The maximum thrust and power associated with the simulated result occur at $0.26 \mathrm{~s}$ with values of $1.17 \mathrm{~N}$ and $22.2 \mathrm{~W}$, respectively. The preceding comparison between measured and simulated motion of the hopping rotochute show that the reported dynamic simulation replicates the major dynamic features of the physical system and is sufficiently accurate to predict performance trends and characteristics as a function of design parameters.

\section{Basic Flight Performance Results}

Using the preceding dynamic model, simulations were performed to determine the basic flight performance of the hopping rotochute. During the first simulation, a series of hops was simulated to assess the maneuverability of the hopping rotochute using a $6 \mathrm{~g}$ internal mass with an offset of $6 \mathrm{~cm}\left(d_{\mathrm{IM}}=6 \mathrm{~cm}\right)$ for directional control. The hop sequence consisted of four hops, each with different internal mass orientations and rotor speed profiles all lasting $1 \mathrm{~s}$ in duration (pulse width $=w_{p}=1 \mathrm{~s}$ ). Before each hop was initiated, the internal mass was oriented to the desired direction and held at this orientation with respect to the body for the duration of the hop. The internal mass was first oriented along the $\vec{I}_{I}$ axis and the rotor speed was ramped up to $2800 \mathrm{rpm}$. Subsequently, the internal mass was oriented along the $-\vec{J}_{I},-\vec{I}_{I}$, and $\vec{J}_{I}$ axes while the rotor speed was ramped up to 3200,3600 , and $4000 \mathrm{rpm}$ for the second, third, and fourth hops, respectively. The resulting trajectory is shown in Fig. 20, which plots the position of the mass center in 3-dimensional space. As shown, the orientation of the internal mass determines the direction of travel, which is caused by the initial launch angle

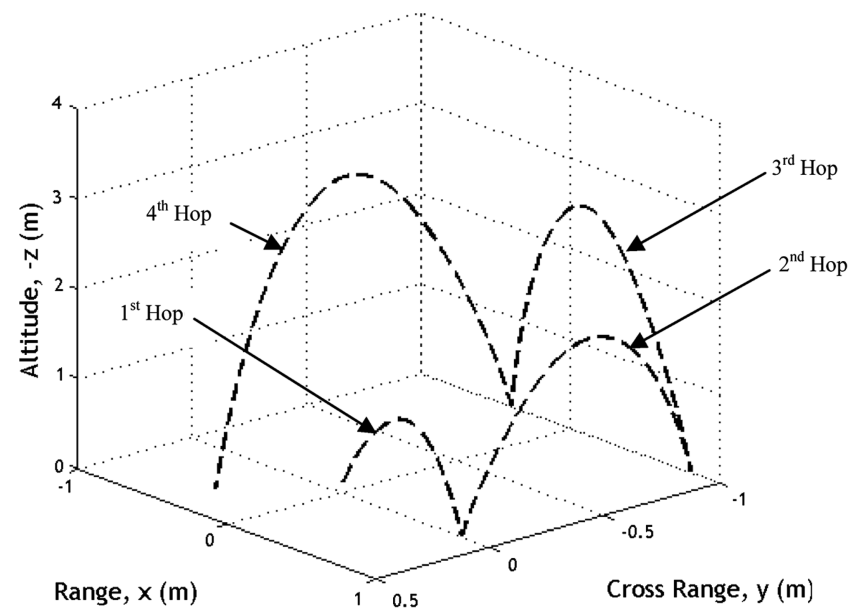

Fig. 20 Altitude versus cross range versus range for a hop sequence.

$(1.32 \mathrm{deg})$ and pitching moment due to the center of mass and rotor thrust axis offset. Figure 20 also demonstrates that the maximum achievable altitude and horizontal distance is increased as the rotor speed is increased. The hopping rotochute is able to climb to a maximum altitude of $1.07,1.83,2.55$, and $3.11 \mathrm{~m}$ while achieving a horizontal distance of $0.82,1.00,1.20$, and $1.31 \mathrm{~m}$ during the first, second, third, and fourth hops, respectively. Although increasing the rotor speed increases the maximum altitude and horizontal distance, more energy is drawn from the battery. As a consequence, the number of hops and total achievable range that can be achieved by the hopping rotochute is driven by the hop profile that is used. To approximate these values, the current versus time curve is numerically integrated to determine the amount of battery discharge that occurred during each of the hops. The number of hops is estimated by dividing the battery capacity $(250 \mathrm{mAh})$ by the discharge associated with a single hop. The total achievable range is then calculated by multiplying the total number of hops by the horizontal distance traveled during each individual hop. Based on this analysis, the hopping rotochute would be able to hop 289 times while traveling up to $237 \mathrm{~m}$ using the hop profile given by the first hop described previously. Using the second, third, and fourth hop profiles would result in a total number of hops of 245,214, and 191 while achieving a total range of 245,257 , and $250 \mathrm{~m}$, respectively.

Because of the fact that the hopping rotochute is powered by a rotor system, even greater altitudes can be achieved by powering the rotor system for extended periods of time. Using the preceding dynamic model, a trade study was performed to determine the maximum achievable altitude as a function of the rotor speed and pulse width $w_{p}$. The results of this trade study are shown in Fig. 21 , in which mass

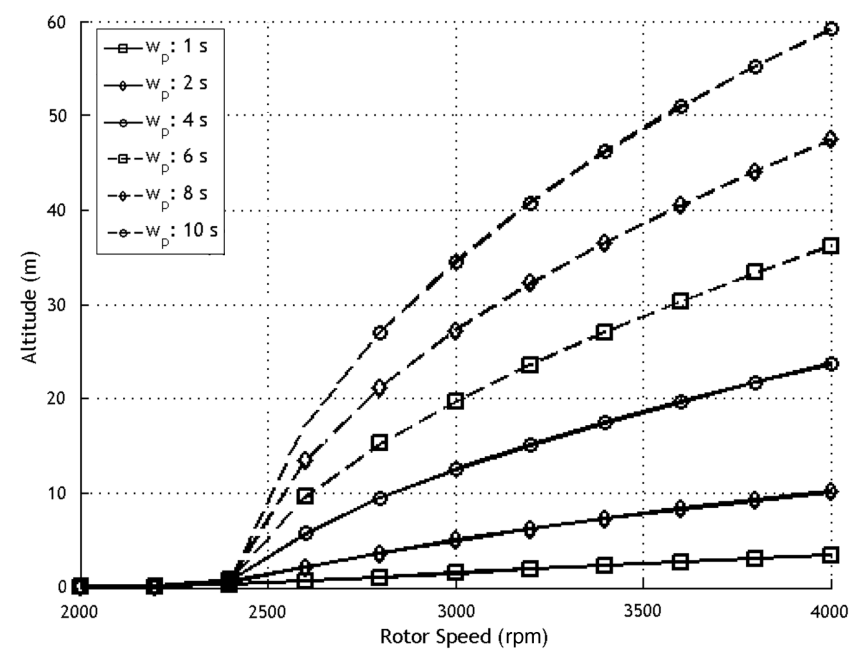

Fig. 21 Altitude versus rotor speed for various pulse widths. 
properties outlined in Table 1 were used. As shown, the maximum altitude increases as the rotor speed and pulse width are increased. In fact, the hopping rotochute is able to climb to an altitude of $60 \mathrm{~m}$ with a rotor speed of $4000 \mathrm{rpm}$ and a pulse width of $10 \mathrm{~s}$.

\section{Conclusions}

The work reported here explores the basic dynamic behavior in flight and on the ground of a new micro hybrid vehicle. This unique vehicle was specifically created to robustly traverse small spaces with complex irregular terrain through a sequence of hops achieved by intermittently powering a coaxial rotor. A dynamic model was developed to predict in-flight and on-ground motions in an integrated fashion. A comparison was made between motion measured using a 12-camera motion capture system and simulation results to assess the validity of the dynamic model. Measured and predicted motions during ground contact and flight agree favorably with the main differences being the horizontal distance traveled during ground impact and the achieved attitude during flight. These differences are mainly attributed to imperfections incurred during fabrication of the device, unaccounted for nonlinear spring and damper constants, and unmodeled rotor dynamics during flight.

By extending the measured results using the simulation model, the practical utility of the hopping rotochute prototype has been highlighted. The hopping rotochute prototype can execute about 250 individual hops and achieve a total range of about $250 \mathrm{~m}$ on a single battery charge. Because power is predominantly used when the rotor is engaged, the vehicle is capable of extended time on station by hopping into a desirable location and remaining stationary to effectively perch and stare for long periods of time. By altering the duration and rotational speed profile of a rotor power pulse, flight characteristics can be greatly modified such that it is possible to shape individual trajectories.

\section{References}

[1] Stoeter, S. A., Rybski, P. E., Gini, M., and Papanikolopoulos, N., "Autonomous Stair-Hopping with Scout Robots,," Proceedings of the IEEE International Conference on Intelligent Robots and Systems, Vol. 1, Inst. of Electrical and Electronics Engineers, Piscataway, NJ, 2002, pp. 721-726.

[2] Morrey, J. M., Lambrecht, B., Horchler, A. D., Riztmann, R. E., and Quinn, R. D., "Highly Mobile and Robust Small Quadruped Robots," Proceedings of the IEEE International Conference on Intelligent Robots and Systems, Vol. 1, Inst. of Electrical and Electronics Engineers, Piscataway, NJ, Oct. 2003, pp. 82-87.

[3] Horchler, A. D., Quinn, R. D., Lambrecht, B., and Morrey, J. M., "Highly Mobile Robots that Run and Jump," U.S. Patent 7249640, July 2007.

[4] Fiorini, P., and Burdick, J., "The Development of the Hopping Capabilities for Small Robots," Autonomous Robots, Vol. 14, No. 2-3, Mar. 2003, pp. 239-254. doi:10.1023/A:1022239904879

[5] Weiss, P., "Hop. ..Hop. ..Hopbots!: Designers of Small, Mobile Robots Takes Cues from Grasshoppers and Frogs," Science News, Vol. 159, No. 6, 2001, pp. 88-90. doi: $10.2307 / 3981566$

[6] Spletzer, B. L., Fischer, G. J., Marron, L. C., Martinez, M. A., Kuehl, M. A., and Feddema, J. T., "Hopping Robot," U.S. Patent 6247546 , June 2001.

[7] Etkin B., Dynamics of Atmospheric Flight, Dover, New York, 2000, pp. 121-152.

[8] Goyal, S., Pinson, E., and Sinden, F., "Simulation of Dynamics of Interacting Rigid Bodies Including Friction 1: General Problem and Contact Model," Engineering with Computers, Vol. 10, No. 3, Sept. 1994, pp. 162-174. doi:10.1007/BF01198742

[9] Goyal, S., Pinson, E., and Sinden, F., "Simulation of Dynamics of Interacting Rigid Bodies Including Friction 2: System Design and Implementation," Engineering with Computers, Vol. 10, No. 3, Sept. 1994, pp. 175-195. doi:10.1007/BF01198743 